\title{
The Classical Theory of Univalent Functions and Quasistatic Crack Propagation.
}

\author{
Gerardo E. Oleaga \\ Departamento de Matemática Aplicada \\ Facultad de Matemáticas, Universidad Complutense de Madrid \\ Ciudad Universitaria s/n 28040, Madrid (Spain) \\ email: oleaga@mat.ucm.es
}

13th March 2006

\begin{abstract}
We study the propagation of a crack in critical equilibrium for a brittle material in a Mode III field. The energy variations for small virtual extensions of the crack are handled in a novel way: the amount of energy released is written as a functional over a family of univalent functions on the upper half plane. Classical techniques developed in connection to the Bieberbach Conjecture are used to quantify the energy-shape relationship. By means of a special family of trial paths generated by the so-called Löwner equation we impose a stability condition on the field which derives in a local crack propagation criterion. We called this the anti-symmetry principle, being closely related to the well known symmetry principle for the in-plane fields.
\end{abstract}

Keywords Crack propagation, Mode III, Univalent functions, Loewner equation, Schiffer's method. 2000 Mathematics Subject Classification 74R10, 74B05, 74G70, 30C55, 30C70.

\section{Introduction}

In the seminal work of A.Griffith the problem of crack stability in a brittle material was handled from first principles of mechanics and thermodynamics (see [12]). The revolutionary idea that he proposed was to apply an energy balance approach instead of the more popular "stress threshold" criterion. Nevertheless, the question of crack evolution was not studied by him and a large amount of research has been carried out to understand this phenomenon from the Engineering, Physical and, more recently, Mathematical viewpoints (cf. for instance [10], [5], [7], [8] and [2]).

The well-posedness of the problem demands at least two scalar equations to find the path of a crack in a two-dimensional setting. Griffith's condition provides only one of them, representing the balance between elastic energy released and crack surface energy dissipated during tip advance. We recall here the asymptotic expansion of the near-tip displacement for an out-of-plane field:

$$
u\left(x_{1}, x_{2}\right)=k_{0}+k_{1} r^{1 / 2} \sin (\theta / 2)+k_{2} r \cos (\theta)+O\left(r^{3 / 2}\right) \quad r \rightarrow 0 .
$$

Here $r, \theta$ denote polar coordinates in the plane $x_{1}, x_{2}$ and we assume that the crack occupies the half line $\left(x_{1}, 0\right)$ for $x_{1} \leq 0$ (see [9] for details). The coefficient $k_{1}$ here is, up to a multiplicative constant, the so-called stress intensity factor in Mode III denoted by $K_{\text {III }}$ in the literature. Notice that expansion (1.1) may be carried out at any stage of the propagation process after a suitable rotation and translation of the current configuration, assuming that the portion of the crack path near the tip is fairly smooth. The local effect of the far applied loads and body geometry is contained in the factors $k_{i}$. In this way, a functional relationship involving the values of the $k_{i}$ 's is interpreted as a crack propagation law, since it imposes a constraint in the way that boundary conditions, crack configuration and near-tip field interact. In this context, Griffith's condition for crack advance may be recalled as follows:

$$
k_{1}=\text { material constant } .
$$


If $k_{1}$ does not reach the threshold value there is no possible crack advance. On the other hand, when condition (1.2) is satisfied, the crack-body configuration is in a critical equilibrium state.

The main purpose of this article is to derive a second explicit condition on the elastic field around the tip to determine the path geometry as a free boundary problem. It turns out that

$$
k_{2}=0,
$$

gives the additional constraint when a stability criterion is imposed for each configuration of the crack. For the in-plane field (Modes I and II) there is a similar relationship known as the symmetry principle (cf. [11], [3], [18], [10]). Nevertheless, the extension of this principle to out-of-plane fields is not obvious.

A secondary but also important aim of this work is to show a remarkable relationship between the stored elastic energy functional in a Mode III field and some classical techniques in Complex Analysis connected to the Bieberbach Conjecture. Among other things, we show that the elastic energy released while a small arbitrary extension of the crack is created can be approximated by a functional defined on a compact set of univalent functions on the upper half plane. This allows to apply Schiffer's method of boundary variation (cf. [4], Appendix) and Löwner evolutions of slit maps (see for instance [6]) to find suitable trial paths. These tools were previously applied in the context of fluid mechanics to other free boundary problems, in particular to study a slit-like morphology for Hele-Shaw flow with suction in the absence of surface tension (see [15, 13]). The methods of Univalent Functions for some free boundary problems in fluid mechanics were brought together more recently in [25], including the analysis of twodimensional solidification/melting of a nucleus in a forced flow. We briefly describe our basic ideas in the following paragraphs and refer to [21] for a discrete version of the model, where we also discuss other approaches to this subject.

Without oversimplifying the vast literature on Linear Elastic Fracture Mechanics we can state that the quasi-static propagation of a crack in a brittle material is performed under the interaction of two physical processes, namely dissipation (irreversible crack surface creation) and elastic deformation (reversible displacements). In this context, dissipation is connected to the work done while breaking atomic bonds during crack advance and it is proportional to the length of the path (see [12], a classical reference). The elastic potential provides the energy that goes into dissipation during crack advance. Its variations with respect to crack extension give birth to the related concept of Energy Release Rate, sometimes called Crack Driving Force.

The dissipative force is similar in nature to classical "dry friction". On the other hand, the crack driving force may be interpreted as the "gradient" of a potential energy. An elementary example of this competition is provided by the rubbing of a material particle against a surface in some force field which depends on the instantaneous position of the particle. Even if this case does not take into account the "history of the motion" as we need to do in the crack problem (ie. the stored elastic energy depends on the whole crack-body configuration), it captures the essential features of the interaction between a reversible driving force and a dissipative force which acts at the tip of the crack during propagation.

In our simple setting we have that if $\delta E(\leq 0)$ is the variation of potential energy and $\delta Q(\geq 0)$ is the corresponding amount of dissipation for an arbitrarily small virtual crack extension, the condition of stable equilibrium for the crack configuration is given by the following inequality:

$$
-\delta E \leq \delta Q \quad \text { for all small enough virtual extensions. }
$$

Notice that $-\delta E:=\delta W$ is the work of the generalized force coming from the potential $E$ and $\delta Q:=\kappa \delta l$, where $\kappa \geq 0$ is a material constant and $\delta l$ is the length of the small crack extension, is the amount of surface energy increment. A more familiar way of writing this condition is $\delta E+\kappa \delta l \geq 0$, which means that the total energy (elastic + surface) should be non-negative while changing from an equilibrium position to a nearby configuration. Strict inequality in (1.4) simply states that if we try to extend the crack a little bit from a stable equilibrium position the amount of work provided by the elastic potential is not enough to cover the dissipation produced by this virtual extensions. If this happens for all small enough trial paths the crack should remain at rest. Condition (1.4) is called "Fourier's inequality" in [17].

In the critical case in which the equality occurs for some trial path,

$$
-\delta E=\kappa \delta l,
$$


we can say that the crack grows quasistatically along a curve in which $-\frac{\delta E}{\delta l}$ is maximum. This corresponds to the well known principle of maximum energy release rate.

The first qualitative conclusion from this formulation is that the shape of the crack is moulded at each stage of propagation by the elastic field around the tip: there is no global optimization function to find the crack configuration as in [8]. In our setting we replace the trial crack shapes by a suitable family of conformal maps. In this way the problem can be tackled using classical techniques of variational calculus for univalent functions. The outcome is a pair of scalar equations (cf. (4.9) and (4.10)) expressing the compatibility of the field with the equilibrium condition of propagation (1.4). As we will see later, condition (4.10) can be called an anti-symmetry principle due to the local field properties it implies. To the author's knowledge, this relationship was not previously considered in the context of out-of-plane fields.

The article is organized as follows. In Section 2 we describe the standard Mode III field equations, the displacement-stress fields, their complex representation and the family of slit maps generated by the crack extensions. This is a straightforward generalization of a former article [21] to arbitrary trial paths. In Section 3 we develope the main technical tools. We introduce the energy functional over a suitable compact family of univalent maps. An application of Schiffer's technique of boundary variations gives a first clue about a suitable selection of trial paths. In Section 4 we introduce Löwner evolutions and, by means of some recent explicit results of W. Kager, B. Nienhuis and L.Kadanoff (cf. [14]), we construct explicit trial paths which give the conditions for the quasistatic evolution of the crack. We conclude with some brief comments about the propagation law obtained.

\section{Basic facts}

\subsection{Out-of-plane fields.}

The Mode III or "out-of-plane" is the simplest of the two-dimensional elastic fields in which the near tip displacement of a crack is resolved (for a general reference see [24]). It is characterized by a single scalar function for the displacement field and two components of stress. To fix ideas consider the initial crack configuration as a half line slit in the plane:

$$
\Gamma_{0}:=\{(x, 0): x \leq 0\}
$$

The elastic body is modelled by the whole plane $\mathbb{R}^{2}$ with the slit $\Gamma_{0}$ deleted. This setting is typical for the computation of the asymptotic field in Mode III when the tip of the crack is "far" from the boundary. The initial displacement field $u_{0}$ is any real function satisfying the following boundary value problem:

$$
\begin{array}{rlrl}
\Delta u_{0} & =0, & x & \in \mathbb{R}^{2} \backslash \Gamma_{0}, \\
\frac{\partial u_{0}}{\partial n}(x) & =0, \quad x \in \Gamma_{0},
\end{array}
$$

where $\Delta=\frac{\partial^{2}}{\partial x_{1}^{2}}+\frac{\partial^{2}}{\partial x_{2}^{2}}$ is the Laplace operator. The Neumann condition (2.2) arises from the fact that there are no given normal tractions along the crack. Additionally, we require the energy condition

$$
\int_{B_{r}(0) \backslash \Gamma_{0}}\left|\nabla u_{0}\right|^{2} d x<\infty, \quad \forall r>0
$$

where $B_{r}(0)$ is the circle of radius $r$ centred at the origin. Inequality (2.3) restricts the behaviour of $u_{0}$ in the vicinity of the crack tip and gives sense to the computation of the energy variation for any crack extension.

We define the evolution of $\Gamma_{0}$ by means of a piecewise smooth curve $\Gamma$ such that

$$
\Gamma_{0} \subset \Gamma \text {. }
$$

The field $u$ corresponding to the boundary given by the extended crack $\Gamma$ will satisfy the equilibrium equation (2.1), the Neumann condition (2.2) and the finite energy condition (2.3) (with $\Gamma_{0}$ replaced by $\Gamma$ 
and $B_{r}(0)$ replaced by $\left.B_{r}(\operatorname{tip}(\Gamma))\right)$. On the other hand, the mapping $u$ is unique once $u_{0}$ and $\Gamma$ are given after imposing a further condition at infinity, which reads as:

$$
\lim _{x \rightarrow \infty}\left|u(x)-u_{0}(x)\right|=0 \quad \text { uniformly in } x .
$$

The uniqueness of the field $u$ is established by conformal mapping of the set $\mathbb{R}^{2} \backslash \Gamma$ into the upper half plane; using Schwarz reflection (applying Neumann homogeneous condition) and concluding by a Liouville-type argument together with (2.4).

It is to be noted that the energy functional

$$
E(u):=\frac{1}{2} \int_{\mathbb{R}^{2} \backslash \Gamma}|\nabla u|^{2}
$$

is not necessarily finite. In spite of this fact, the energy increment given by

$$
\Delta E:=\frac{1}{2} \int_{\Delta \Gamma}[u] \frac{\partial u_{0}}{\partial n^{+}} .
$$

is a finite quantity, where $\Delta \Gamma:=\Gamma \backslash \Gamma_{0},[u]:=u^{+}-u^{-}$is the jump discontinuity of the displacement field over $\Delta \Gamma$, and the superscripts \pm indicate the upper and lower limits of the field on each face of $\Delta \Gamma$. In addition, $\frac{\partial}{\partial n^{+}}$is the normal derivative considering the exterior normal to the upper face of the extended crack (see Figure 1). Notice that (2.6) is valid for finite extensions and for arbitrary shapes of $\Delta \Gamma$. It represents minus the work done by elastic forces when we change the configuration from $\Gamma_{0}$ to $\Gamma$. Notice that we nondimensionalized the energy avoiding a material constant in its definition.

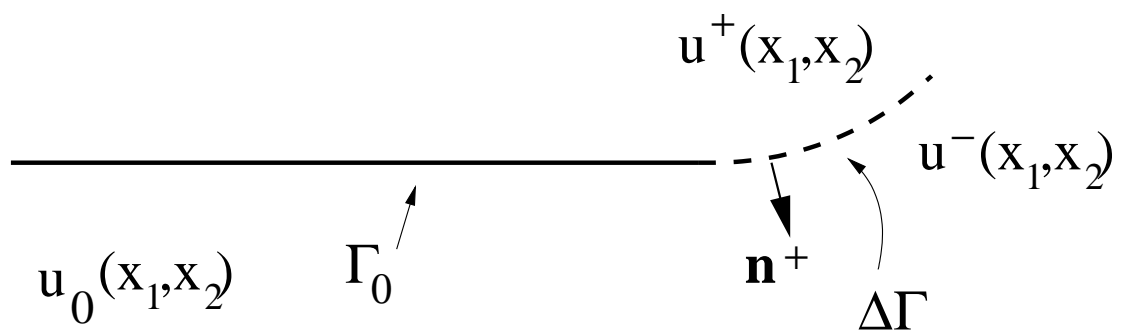

Figure 1: Variables involved in the energy increment formula

\subsection{Complex representation and Slit Maps}

We will denote by $\mathbb{C}$ the set of complex numbers and by $\widehat{\mathbb{C}}:=\mathbb{C} \cup\{\infty\}$ the extended complex plane. $\mathbb{H}$ is the upper half plane $\operatorname{Im} z>0$, and $\widehat{\mathbb{H}}:=\mathbb{H} \cup \mathbb{R}$ the closure of $\mathbb{H}$.

Basic properties of harmonic functions provide the existence a complex function $\eta$, analytic in $\mathbb{C} \backslash \Gamma$, such that:

$$
\operatorname{Re} \eta(\zeta)=u \text {. }
$$

and moreover:

$$
\eta^{\prime}(\zeta)=\frac{\partial u}{\partial x_{1}}-i \frac{\partial u}{\partial x_{2}}, \quad \zeta=x_{1}+i x_{2} .
$$

Assume now that $\Delta \Gamma$ is a Jordan arc (ie. is the continuous image of a finite line interval). By the Riemann Mapping Theorem we can send the set $\mathbb{C} \backslash \Gamma$ onto the upper half plane $\mathbb{H}$ in a one to one conformal correspondence. We will denote by $f: z \in \mathbb{H} \mapsto \zeta \in \mathbb{C} \backslash \Gamma$ the inverse of one of this maps, which admits a continuous extension to the real axis by Carathéodory's Extension Theorem (see [6, p. 12]). For $\Gamma=\Gamma_{0}(\Delta \Gamma=\emptyset)$ we take $f(z) \equiv f_{0}(z):=-z^{2}$. When $\Gamma$ is strictly bigger than $\Gamma_{0}(\Delta \Gamma \neq \emptyset)$ there exists a finite closed interval $I$ on the real line such that (cf. Figure 2):

$$
f^{-1}(\Delta \Gamma)=I
$$



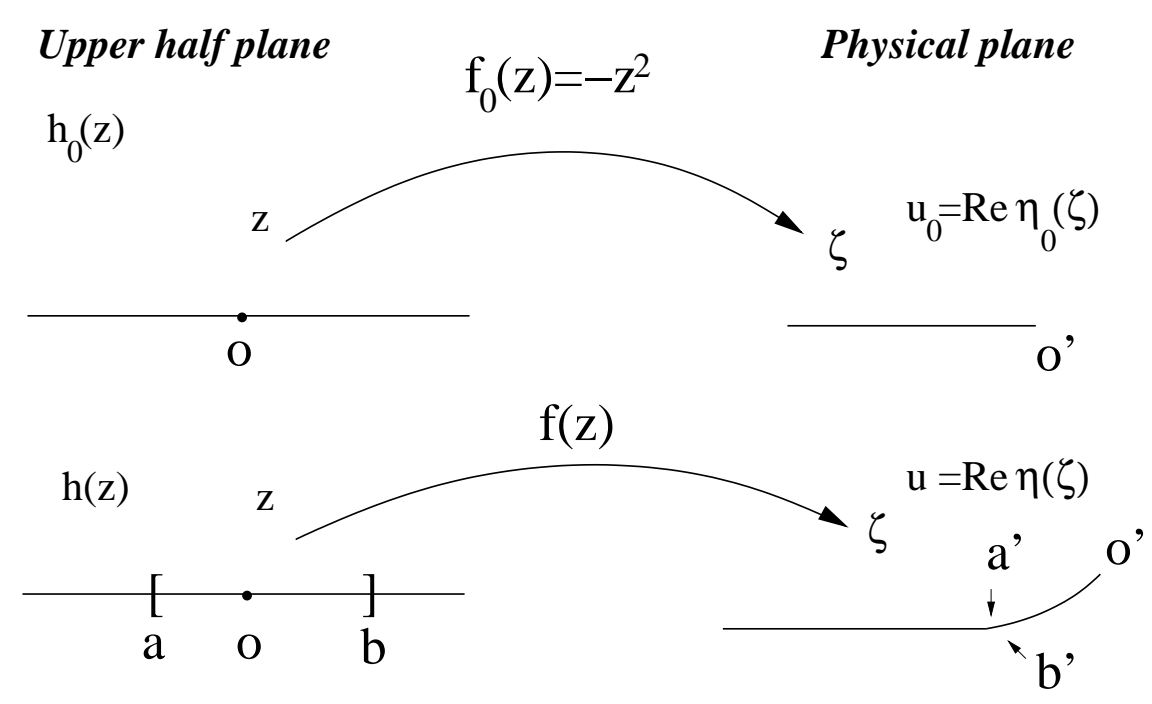

Figure 2: The maps $f_{0}$ and $f$

Consider now the following function:

$$
h(z):=\eta \circ f(z), \quad z \in \mathbb{H} .
$$

We have that

$$
h^{\prime}(z)=\eta^{\prime}(f(z)) f^{\prime}(z)=\left(\frac{\partial u}{\partial t}-i \frac{\partial u}{\partial n}\right)\left|f^{\prime}(z)\right|,
$$

where $(t, n)$ represent the directions that are images through the map $f$ of the axes $\left(z_{1}, z_{2}\right)$ on the $\zeta$ plane. In terms of the function $h$, the boundary conditions take the form

$$
\operatorname{Im} h^{\prime}(z)=0, \quad \text { for } z \in \mathbb{R} .
$$

Applying Schwarz's symmetry principle (cf. [20]) we can extend $h^{\prime}$ (and therefore $h$ ) analytically to the lower half plane by means of

$$
h^{\prime}(z)=\overline{h^{\prime}(\bar{z})} \text {. }
$$

Condition (2.3) for $u$ and $\Gamma$, rules out any singularity around the origin. Notice that

$$
h_{0}(z):=\eta_{0} \circ f_{0}(z) \text {. }
$$

It is possible to write the energy increment given in (2.6) by means of an integral in the complex plane. We can show that

$$
\Delta E \underset{(i)}{=} \frac{1}{2} \int_{I} \operatorname{Re} h(x) \operatorname{Im}\left(\eta_{0} \circ f\right)^{\prime}(x) d x \underset{(i i)}{=} \frac{i}{4} \int_{C} h(z)\left(\eta_{0} \circ f\right)^{\prime}(z) d z,
$$

where $C$ is a closed path surrounding the interval $I$ defined in (2.9). Identity $(i)$ above is obtained with (2.6) after changing variables $\zeta=f(z)$ and taking (2.10) and (2.11) into account. In identity (ii), we used basic properties of complex integration together with the fact that $h(x)$ is real over the real axis and $\eta_{0} \circ f$ is real on $\mathbb{R} \backslash I$. After integrating by parts and taking into account that $\eta_{0}(\zeta)=h_{0} \circ f_{0}^{-1}(\zeta)$ we obtain the following relationship:

$$
\Delta E=\frac{1}{4 i} \int_{C} h^{\prime}(z) h_{0}\left(f_{0}^{-1} \circ f(z)\right) d z .
$$

By (2.12) $h$ is analytic in $\mathbb{C}$ and admits an expansion in the variable $z$ with real coefficients:

$$
h(z)=\sum_{n=0} c_{n} z^{n}, \quad c_{n} \in \mathbb{R} .
$$


Therefore, the following relationship is valid in the original domain:

$$
\eta(\zeta)=\sum_{n=0} c_{n}\left(f^{-1}(\zeta)\right)^{n}, \quad c_{n} \in \mathbb{R}, \zeta \in \mathbb{C} \backslash \Gamma .
$$

Taking the real part this expansion turns out to be:

$$
u\left(x_{1}, x_{2}\right)=\operatorname{Re}\left(\sum_{n=0} c_{n}\left(f^{-1}(\zeta)\right)^{n}\right), \quad x_{1}+i x_{2}=\zeta
$$

Notice that the coefficients $c_{n}$ depend on the configuration of the crack $\Gamma$. In the case $f \equiv f_{0}$, after taking polar coordinates, we have that

$$
u_{0}(r \cos \theta, r \sin \theta)=\operatorname{Re}\left(\sum_{n=0} c_{n}(\sqrt{-\zeta})^{n}\right)=c_{0}-c_{1} r^{1 / 2} \sin (\theta / 2)-c_{2} r \cos (\theta)+\ldots
$$

This is the same expansion as the one in (1.1) where $k_{0}=c_{0}, k_{1}=-c_{1}, k_{2}=-c_{2}, \ldots$

Our next purpose is to write the functional $\Delta E$ given in (2.15) in terms of the coefficients $c_{n}$ and the mapping functions $f_{0}, f$. To this end it is more convenient to consider the map:

$$
F(z):=f_{0}^{-1} \circ f(z)=\sqrt{-f(z)},
$$

carrying $\mathbb{H}$ to the set $\mathbb{H} \backslash \gamma$, where $\gamma:=\sqrt{-\Delta \Gamma}$ is a finite Jordan arc lying in $\mathbb{H}$ with one tip attached to the origin (see Figure 3 below).

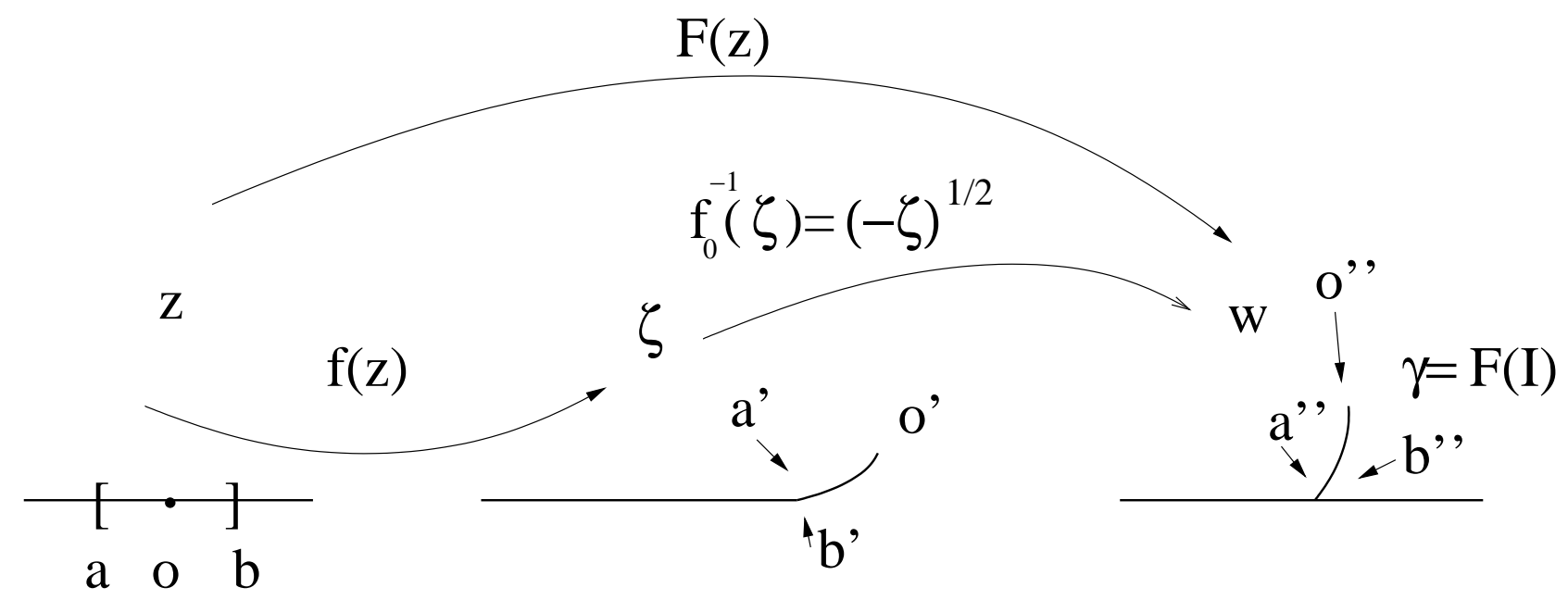

Figure 3: The map $F$

The map $F$ takes real values on $\mathbb{R} \backslash I$ and it can be extended to the whole plane minus $I$ by Schwarz reflection, ie. $F(z)=\overline{F(\bar{z})}$. We can take the function $f$ (after a suitable change of scale in $z$ ) such that $F$ admits an expansion of the form

$$
F(z ; \gamma)=z+b_{0}(\gamma)+\frac{b_{1}(\gamma)}{z}+\ldots \quad|z|>Z, b_{i} \in \mathbb{R}
$$

for some $Z>0$. We recall that $F$ should be the identity for $\Gamma=\Gamma_{0}$ (ie., when $\gamma=\emptyset$ and $I=\emptyset$ ). Notice that the location of the interval $I$ is somehow arbitrary, and therefore the mapping $F$ is not uniquely defined for a given curve $\gamma$. We denote by $c_{n}(\gamma)$ the coefficients appearing in (2.16) for $\Delta \Gamma \neq \emptyset$ and simply $c_{n}$ when $\Delta \Gamma=\emptyset(\gamma=\emptyset)$. We can then write, using (2.16) and (2.20) inside (2.15), the following relationship:

$$
\Delta E=\frac{1}{4 i} \int_{C}\left(\sum_{j=1} j c_{j}(\gamma) z^{j-1}\right)\left(\sum_{k=0} c_{k}(F(z ; \gamma))^{k}\right) d z .
$$


The functional $\Delta E$ depends on the initial field and on the curve $\gamma$ which in turn defines a conformal map $F$ and an interval $I$ (cf.Figure 3). The coefficients $c_{n}$ depend explicitly on the shape of the growing crack. This dependence is explicitly shown in the following Proposition.

Proposition 1 The coefficients $c_{n}(\gamma)$ in (2.22) satisfy the following relationship:

$$
c_{n}(\gamma)=\frac{1}{2 \pi i} \sum_{j=n}^{\infty} c_{j} \int_{C} \frac{F(z ; \gamma)^{j}}{z^{n+1}} d z
$$

where $F:=f_{0}^{-1} \circ f$ is given in (2.21) and $C$ is a closed curve surrounding the interval I of Figure 3.

Proof. The real function given by

$$
v(x):=u(x)-u_{0}(x)
$$

is harmonic in $\mathbb{C} \backslash \Gamma$. On the other hand, by the matching condition (2.4) it goes to zero uniformly at infinity. By (2.2) it satisfies homogeneous Neumann boundary conditions on $\Gamma_{0}$ and

$$
\frac{\partial v}{\partial n}(x)=-\frac{\partial u_{0}}{\partial n}(x) \quad x \in \Delta \Gamma
$$

We have that: (a) $v(f(z))$ is harmonic in the upper half plane, (b) it goes to zero at infinity, (c) satisfies homogeneous Neumann boundary conditions on $\mathbb{R} \backslash I$ and non homogeneous Neumann conditions on $I$. By (c) it can be extended by symmetry to a harmonic function in $\mathbb{C} \backslash I$. Using the analytic completion of the Poisson formula for the upper half plane we have that:

$$
v(f(z))=\operatorname{Re} p(z),
$$

where $p$ is holomorphic in $\widehat{\mathbb{C}} \backslash I$ (recall that $\widehat{\mathbb{C}}:=\mathbb{C} \cup\{\infty\}$ ) and it can be taken such that $p(\infty)=0$. It therefore admits a Laurent expansion for $|z|>R$, where $I \subset[-R, R]$, with strictly negative powers of $z$. We have then:

$$
h(z)=g(z)+p(z),
$$

where $g(z):=\eta_{0}(f(z))$ (cf. 2.10 and 2.13). As $h$ is analytic in the whole $\mathbb{C}$, we must have that $g(z)$ and $p(z)$ share the negative powers in $z$ (with coefficients of opposite sign) in their respective Laurent expansions around $z=0$. Therefore, if the curve $C$ encloses $z$ and $I$, we must have that:

$$
h(z)=\frac{1}{2 \pi i} \int_{C} \frac{g(\zeta)}{\zeta-z} d \zeta
$$

The function $g(z)$ may be written as follows:

$$
g(z)=\eta_{0}(f(z))=h_{0}\left(f_{0}^{-1} \circ f(z)\right)=h_{0}(F(z ; \gamma)) .
$$

We recall that the function $F$ sends the upper half plane in a one to one way to the upper half plane minus the set $\gamma:=f_{0}^{-1}(\Delta \Gamma)$, taking real values on $\mathbb{R} \backslash I$. Therefore, it can be extended by symmetry to an analytic univalent function on $\mathbb{C} \backslash I$ and admits an expansion of the form (2.21). We use now the formula for the coefficients in a Laurent expansion (cf. [20]), taking into account the development of $h_{0}$ given in (2.16) and (2.24), obtaining:

$$
c_{n}(\gamma)=\frac{1}{2 \pi i} \int_{C} \frac{g(z)}{z^{n+1}} d z=\frac{1}{2 \pi i} \int_{C} \frac{h_{0}(F(z ; \gamma))}{z^{n+1}} d z=\frac{1}{2 \pi i} \sum_{j=0}^{\infty} c_{j} \int_{C} \frac{F(z ; \gamma)^{j}}{z^{n+1}} d z
$$

We replace now $F(z ; \gamma)$ using $(2.21)$ :

$$
c_{n}(\gamma)=\frac{1}{2 \pi i} \sum_{j=0}^{\infty} c_{j} \int_{C} \frac{d z}{z^{n+1}}\left(z+\sum_{k=0}^{\infty} \frac{b_{k}(\gamma)}{z^{k}}\right)^{j} .
$$


We see at once that:

$$
\int_{C} \frac{d z}{z^{n+1}}\left(z+\sum_{k=0}^{\infty} \frac{b_{k}(\gamma)}{z^{k}}\right)^{j}=0 \text { for } j<n
$$

and then we can write:

$$
c_{n}(\gamma)=\frac{1}{2 \pi i} \sum_{j=n}^{\infty} c_{j} \int_{C} \frac{d z}{z^{n+1}}\left(z+\sum_{k=0}^{\infty} \frac{b_{k}(\gamma)}{z^{k}}\right)^{j}
$$

which is (2.23).

\section{Univalent functions on $\mathbb{H}$.}

\subsection{Motivation for this Section}

Fourier's inequality (1.4) and the critical growth condition (1.5) imply that the crack will try to find a compromise between a maximum amount of elastic energy released by the body and a minimum of energy dissipated in the crack opening. In this Section we explore the optimal shape for maximum energy released in a set of crack extensions of limited size. In first place we consider the main term in the energy release expansion and later we include the first two tems in (2.22). As we will see, it is possible to write the energy released as a functional over the set of univalent functions on the upper half plane. In the following Section we will use the optimal shape obtained as a "trial path" to obtain explicit conditions for the field surrounding the free boundary.

\subsection{A set of conformal maps as trial paths.}

In Section 2.2 we considered an initial crack $\Gamma_{0}$ and its extension $\Delta \Gamma$. In the new configuration $\Gamma:=\Gamma_{0} \cup \Delta \Gamma$ we used a conformal map $f$ (not unique) to pose the problem in the upper half plane $\mathbb{H}$. With the help of classical properties of analytic functions and Neumann boundary conditions, we defined a functional $\Delta E$ measuring the amount of energy released by the appearance of $\Delta \Gamma$ (cf. 2.22). This approach is not yet complete for our purposes. We want to determine an optimal $\Delta \Gamma$ but we still do not have a suitable set of curves in which this shape can be found. Moreover, we did not define an appropriate topology on the space of admissible paths; therefore the continuity of the functional $\Delta E$ is, right now, an empty statement.

In this Section we change the point of view: we will focus on the conformal maps instead of the sets defining the crack extension. Taking the function $f$ as above, we define a map $F:=\sqrt{-f(z)}$, with $F: \mathbb{H} \mapsto \mathbb{H}$. There exists an interval $I \subset \mathbb{R}$ such that the extension by symmetry of $F$ has real values on $\mathbb{R} \backslash I$ and $F(I)=\gamma$ (cf. Figure 3). Instead of focusing on the curve $\gamma$ as a variable, we drive now our attention to a family of univalent maps (cf. [6]) defined on the upper half plane $\mathbb{H}$ and taking values on $\mathbb{H}$. A closer look at the formulae for $\Delta E$ and $c_{n}$ in (2.22) and (2.23) reveals that the functional dependence of $\Delta E$ on $\gamma$ is explicit through the mapping $F$. The growing crack can be visualized as the set of omitted values of this conformal map after a suitable transformation, ie. $\gamma=\mathcal{O}:=\mathbb{H} \backslash F(\mathbb{H})$. Taking as a guide the properties of the functions already considered in Section 2.2, we will define a proper setting where we can look for the desired path. There are two main assumptions that we must keep in mind.

In the first place we must find the more relevant terms in the released energy for small extensions of the crack. We must then control the size of the set of omitted values. We will see that, after a suitable normalization of the behaviour of the map $F$ at infinity, the size of $\mathcal{O}$ is controlled by the length of the interval $I$ (cf. Figure 3). An easy way to guess this relationship consists in applying the following change of scale to the map $F$ :

$$
\widetilde{F}(z)=\lambda F\left(\frac{z}{\lambda}\right) .
$$

If $F$ corresponds to a single slit $\gamma$ departing from the origin and is normalised as in (2.21) it is easy to check that $\widetilde{F} \sim z$ as $z \rightarrow \infty$, and corresponds to the slit $\lambda \gamma$ with an interval $\lambda I$. If we allow $I$ to be unbounded then we can take $\lambda>0$ as big as we want to produce arbitrarily "big" cracks. Nevertheless, this is not the true physical constraint to the trial paths since the correct way to control the energy dissipated is through 
the actual crack length. We will temporarily use the size of $I$ as an artificial constraint to guess the shape of the optimal growing path. Later we will come back to this point through Löwner evolutions.

In the second place, it is necessary to fix a full normalization for the maps $F$ to obtain a suitable expansion for the released energy. We must be sure that when we change the slit $\gamma$ the corresponding map measures the sets under the same scale. In order to have a unique representation for each omitted set we choose the so-called hydrodynamic normalization (see point 2 in Definition below).

We are now in a position to define the family of transformations that describe the admissible competitors for the optimal crack shape.

Definition 2 Given $R>0$, let $\mathcal{F}_{R}$ be the family of univalent functions $F: \mathbb{H} \mapsto \mathbb{H}$ satisfying the following additional conditions:

1. $F(x) \in \mathbb{R}$ for $x \in \mathbb{R}$ such that $|x|>2 R$.

2. $F(\infty)=\infty$ with the following expansion

$$
F(z)=z+\frac{b_{1}}{z}+\frac{b_{2}}{z^{2}} \ldots, \quad|z|>2 R
$$

It is possible to extend $F$ by symmetry to $\mathbb{C} \backslash I$. The resultant function is still univalued on the points of analyticity being a sectionally analytic function (ie. $F$ is analytic out of the interval $I:=[-2 R, 2 R]$ ). In the following, we will identify $F$ with its extension. Notice also that in property 2 of Definition 2 we set $b_{0}=0$ (cf. 2.21).

Proposition $3 \mathcal{F}_{R}$ is compact in the topology of uniform convergence of functions over compact sets and the following bound is valid for the coefficients in expansion (3.1):

$$
\left|b_{n}\right| \leq n^{-1 / 2}(2 R)^{n+1}, \quad n=1,2, \ldots
$$

Proof. The bound (3.2) for $n \geq 1$ is a consequence of the classical Area Theorem (see [6], Corollary to Theorem 2.1 and bottom of page 326). We must only take into account that $F$, extended to the lower half plane, is analytic on $\mathbb{C} \backslash I$, and after property 1 ) in Definition 2 is univalent outside a disc of radius $2 R$.

We turn now to the compactness property. By Montel's Theorem (cf. [23], page 35) we show first that $\mathcal{F}_{R}$ is normal by proving that the family is locally bounded. Consider the map

$$
T_{R}(w):=w+\frac{R^{2}}{w},
$$

which sends the exterior of the circle of radius $R$ onto the complement of the slit $[-2 R, 2 R]$. We have that $G(w)=F\left(T_{R}(w)\right)$ is a univalent map defined on the complement of the disc of radius $R$ with the following expansion around infinity:

$$
G(w)=w+\frac{R^{2}+b_{1}}{w}+O\left(\frac{1}{w^{2}}\right), \quad|w|>R .
$$

By Lemma 5.1.3 in [23], the following growth condition is valid for $G$ :

$$
|G(w)| \leq 2|w| \text { for }|w|>R,
$$

and the condition for $F$ is given by the following inequality:

$$
|F(z)| \leq 2\left|T_{R}^{-1}(z)\right| \quad \text { for } z \in \mathbb{C} \backslash[-2 R, 2 R] .
$$

We conclude that the whole family is locally bounded and $\mathcal{F}_{R}$ is normal on $\mathbb{C} \backslash[-2 R, 2 R]$ by Montel's theorem.

The compactness of $\mathcal{F}_{R}$ follows from the following properties: a) the fact that analyticity and univalence are preserved under uniform convergence over compact sets (see [6] page 5, together with the fact that 
the representation (3.1) precludes a constant limit), and b) that the properties 1) and 2) of Definition 2 are preserved in the limit. We will make further comments about this last statement. If $F_{n}(z) \in \mathbb{H}$ and $F_{n}(z) \rightarrow F(z)$ for $z \in \mathbb{H}$, then $F(z) \in \mathbb{H}:$ Assume that this is not the case; then there exists some $z \in \mathbb{H}$ such that $\operatorname{Im} F(z) \leq 0$. Due to the univalency of $F$, we must have that the image of a small disc around $z$ contains a disc around $F(z)$ (open mapping theorem). Then there must be some $\hat{z}$ with $\operatorname{Im} \hat{z}>0$ such that $\operatorname{Im} F(\hat{z})<0$, this contradicts the fact that $F$ is a uniform limit of functions $F_{n}: \mathbb{H} \mapsto \mathbb{H}$. Property 1 ) is a consequence of the fact that pointwise limit of real values is also real for $x \in \mathbb{R} \backslash I$. Property 2) is a consequence of Cauchy's formula for the coefficients together with the property of uniform convergence over compact sets.

We have now all the ingredients to look for an optimal shape inside the compact family $\mathcal{F}_{R}$.

\subsection{The Energy functional}

Let us recall the expression for the released energy in terms of the mapping function $F$. We replace the dependence on $\gamma$ in (2.22) by the map $F$ :

$$
\Delta E=\frac{1}{4 i} \int_{C}\left(\sum_{j=1}^{\infty} j c_{j}(F) z^{j-1}\right)\left(\sum_{k=0}^{\infty} c_{k} F(z)^{k}\right) d z .
$$

Our aim is to maximize $-\Delta E$ for $F$ in the normalized family $\mathcal{F}_{R}$. The integration path should surround the interval $[-2 R, 2 R]$. Now we take into account the result of Proposition 1 together with the bounds for the $b_{i}$ 's (3.2) obtained in Proposition 3. We can write

$$
c_{n}(F)=c_{n}+(n+1) c_{n+1} b_{0}(F)+O\left(R^{2}\right), \quad R \rightarrow 0
$$

where $c_{n} \equiv c_{n}$ (Id) refers (as before) to the coefficients of the displacement field when $F(z)=z$ (ie, no added crack). Therefore, it is possible to write the following expansion for the energy released:

$$
\begin{aligned}
\Delta E & =\frac{1}{4 i} \int_{C}\left(c_{1}^{2} F(z)+c_{1} c_{2}\left(F(z)^{2}+2 F(z)\left(z+b_{0}(F)\right)\right)\right) d z+O\left(R^{4}\right) \\
& =\frac{\pi}{2}\left(c_{1}^{2} b_{1}(F)+4 c_{1} c_{2}\left(b_{0}(F) b_{1}(F)+b_{2}(F)\right)\right)+O\left(R^{4}\right), \quad R \rightarrow 0 .
\end{aligned}
$$

The terms considered correspond to the index values $(j, k)=(1,1),(1,2)$ and $(2,1)$ in equation $(2.22)$. By property (2) of Definition 2, we have that $b_{0}(F) \equiv 0$. Then we will take, as an approximation to the shape problem for $R \rightarrow 0^{+}$, the following energy functional over the family $\mathcal{F}_{R}$ :

$$
\mathcal{E}(F):=\frac{\pi}{2}\left(c_{1}^{2} b_{1}(F)+4 c_{1} c_{2} b_{2}(F)\right) .
$$

Notice that $c_{0}$ has no influence on the energy increment. The main contribution to $\mathcal{E}$ as $R \rightarrow 0^{+}$is given by the term involving the coefficient $b_{1}(F)$. This corresponds to the so-called Energy Release Rate in Fracture Mechanics and will be considered in the first place. This term is of order $R^{2}$ by (3.2). For maps $F$ generating "kinks" we have that $R$ is of the order of $\sqrt{l}, l$ being the length of the kink (cf. [21]). In this case the quantity $\lim _{R \rightarrow 0^{+}} \frac{c_{1}^{2}}{R^{2}} b_{1}(F)$ is related to the energy released per unit length of the growing crack (leaving aside multiplicative constants).

\subsection{The best shape for optimal energy release rate.}

Let us approximate the functional $\mathcal{E}$ in (3.7) neglecting the term $4 c_{1} c_{2} b_{2}(F)=O\left(R^{3}\right)$ (cf. (3.2)):

$$
\mathcal{E}(F) \approx \frac{\pi}{2} c_{1}^{2} b_{1}(F)
$$

In order to maximize $-\mathcal{E}$, we must find the mapping $F \in \mathcal{F}_{R}$ that provides the minimum value for the coefficient $b_{1}$. This minimum is attained due to the compactness of $\mathcal{F}_{R}$ and the continuity of the functional:

$$
b_{1}(F), \quad F \in \mathcal{F}_{R} .
$$


In order to find the optimal $F$ we make use of the elementary theory of univalent functions (cf. [6]). Consider the map $T_{R}$ given in (3.3), the function $G(w)=F\left(T_{R}(w)\right)$ and its expansion at $w=\infty$ given in (3.4). By an application of the Area Theorem to $G(w)$ (in the same spirit that for the bound (3.2)) we have that $\left|R^{2}+b_{1}\right| \leq R^{2}$, and the equality holds if and only if $G$ is given by (see [6], Corollary to Theorem 2.1 therein)

$$
G(w)=w+\frac{d_{1}}{w}, \quad\left|d_{1}\right|=R^{2} .
$$

Therefore, the minimum value of $d_{1}$ corresponds to $-R^{2}$, and then $b_{1}=-2 R^{2}$ should be also a minimum. The composition with the map $T_{R}^{-1}$,

$$
w=T_{R}^{-1}(z)=\frac{z+\sqrt{z^{2}-4 R^{2}}}{2},
$$

sending the set $\mathbb{C} \backslash[-2 R, 2 R]$ on $\mathbb{C} \backslash(R \mathbb{D})$, gives the function $F$ :

$$
F(z)=G\left(T_{R}^{-1}(z)\right)=\frac{z+\sqrt{z^{2}-4 R^{2}}}{2}-\frac{2 R^{2}}{\left(z+\sqrt{z^{2}-4 R^{2}}\right)}
$$

After some manipulation of this expression, we obtain that

$$
F(z)=\sqrt{z^{2}-4 R^{2}}
$$

is the unique optimal map in $\mathcal{F}_{R}$. This corresponds to a straight vertical slit in the upper half plane, from $z=0$ to $z=i 2 R$. If we go back to the original domain through the map $z \rightarrow-z^{2}$, we have that

$$
z \in \mathbb{H} \mapsto f(z)=4 R^{2}-z^{2}
$$

is the function defining the optimal shape of the crack for the simplified case at hand. This corresponds to the horizontal segment $\left[0,4 R^{2}\right]$ in the domain of $\Gamma_{0}$ (ie. in the middle of Figure 3 ) for $z$ in the interval $[-2 R, 2 R]$. Thus, the straight line is preferred among a very general family of competitors. In particular this shows that, in this context, the best growing strategy is through a unique slit. In other words, it is energetically more efficient to grow without bifurcation.

\subsection{Schiffer's variational method}

We consider (3.7) in its full form:

$$
\mathcal{E}(F)=\frac{\pi}{2}\left(c_{1}^{2} b_{1}(F)+4 c_{1} c_{2} b_{2}(F)\right)
$$

The maximum of $-\mathcal{E}$ corresponds to the minimum of $\mathcal{E}$ with $c_{1}, c_{2} \in \mathbb{R}$ given. This is a linear function of the coefficients of the map $F$, which are themselves continuous functionals on the compact family $\mathcal{F}_{R}$. Therefore, there exists at least one $F \in \mathcal{F}_{R}$ minimizing $\mathcal{E}$. This kind of problem, arising in connection with the theory of univalent functions, is closely connected to the mathematical tools developed for the proof of the celebrated Bieberbach conjecture. One of the most powerful approaches is the so-called Schiffer's method of boundary variation (see for example his Appendix to Courant's book [4]). The basic idea is to perturb the extremal function by composing it with maps univalent on its range which are close to the identity. Schiffer's Theorem converts the resulting collection of inequalities into the conclusion that the set omitted by an extremal function is a system of analytic arcs which are trajectories of a certain quadratic differential (see also [6]).

Before applying Schiffer's method we need to make some further comments. The functional $\mathcal{E}$ in $(3.7)$ is well defined for the bigger class of univalent maps that are analytic outside the interval $I$. That is, the functional may be evaluated on maps that are not symmetric with respect to the real axis. When $F(\bar{z})=\overline{F(z)}$, the $b_{i}$ 's are real and $\operatorname{Re} \mathcal{E}(F)=\mathcal{E}(F)$. Therefore, we will proceed to minimize the real part of $\mathcal{E}$ over the wider set of univalent maps on $\mathbb{C} \backslash I$ satisfying the hydrodynamic normalization. Later on, 
we will show that the minimizers belong in fact to $\mathcal{F}_{R}$. We follow essentially the book of Duren [6] in our presentation.

Consider a univalent variation of $F$ given by the map

$$
\chi(w)=w+\frac{\lambda_{r}}{w-w_{0}}+o\left(r^{2}\right), \quad r \rightarrow 0^{+},
$$

where $w_{0} \in \mathbb{H}$ belongs to the set of omitted values of $F$ and $\lambda_{r}$ satisfies:

$$
\lim _{r \rightarrow 0^{+}} r^{-2}\left|\lambda_{r}\right|>0
$$

We now evaluate the real part of the functional $\mathcal{E}$ :

$$
\operatorname{Re}\{\mathcal{E}(\chi \circ F)\}-\operatorname{Re}\{\mathcal{E}(F)\}=\lambda_{r} \ell\left(\frac{1}{F-w_{0}} ; F\right)+o\left(r^{2}\right),
$$

where $\ell(\cdot ; F)$ is the Fréchet differential of $\operatorname{Re}\{\mathcal{E}\}$ at $F$. Then we must have (cf. [6])

$$
\operatorname{Re}\left\{\lambda_{r} s\left(w_{0}\right)+o\left(r^{2}\right)\right\} \geq 0
$$

for all $w_{0}$ in the omitted set, where

$$
s\left(w_{0}\right):=\ell\left(\frac{1}{F-w_{0}} ; F\right) .
$$

According to Schiffer's Theorem this leads to the following quadratic differential for the omitted set:

$$
s(w(t))\left(\frac{d w}{d t}\right)^{2}<0 .
$$

In order to compute the trajectories, we must find the explicit expression for $s$. To this end we analyze the changes in the coefficients $b_{1}$ and $b_{2}$ that take place when we perform the variation

$$
\frac{\varepsilon}{F-w}, \quad \text { for }|\varepsilon| \ll 1
$$

We have that:

$$
\frac{d b_{1}}{d \varepsilon}=1, \quad \frac{d b_{2}}{d \varepsilon}=w
$$

Thus

$$
\ell\left(\frac{1}{F-w} ; F\right)=\frac{\pi}{2}\left(c_{1}^{2}+4 c_{1} c_{2} w\right)
$$

The quadratic differential is given now explicitly by the expression

$$
\left(c_{1}^{2}+4 c_{1} c_{2} w\right)\left(\frac{d w}{d t}\right)^{2}<0 .
$$

Or in other terms (selecting a suitable parameterization),

$$
\sqrt{c_{1}^{2}+4 c_{1} c_{2} w} \frac{d w}{d t}=i
$$

Notice that if we impose $c_{2}=0$ (as in the previous case), then (3.10) turns out to be

$$
\left|c_{1}\right| \frac{d w}{d t}=i
$$

This is the equation for straight vertical trajectories. For $w(0)=0$ it gives $w(t)=t i /\left|c_{1}\right|$, and we recover the previous result. 
We proceed to integrate (3.10) assuming that $c_{1} c_{2} \neq 0$ :

$$
\frac{d}{d t}\left(c_{1}^{2}+4 c_{1} c_{2} w\right)^{3 / 2}=6 c_{1} c_{2} i
$$

gives

$$
\left(c_{1}^{2}+4 c_{1} c_{2} w\right)^{3 / 2}=6 c_{1} c_{2} i t+k,
$$

where $k$ is an integration constant that may depend on $R$. If we assume that the optimal path will grow starting from the origin we must have that

$$
k=\left|c_{1}\right|^{3} .
$$

It is not obvious how to justify this assumption within this mathematical context, but we can give several ad-hoc arguments in favor of (3.13). One of them is that the crack cannot initiate away from the origin because there is no other singularity on the elastic field. With no singularity there is no possible driving force to produce a crack extension. Another kind of argument may be called a "continuity argument" and it is as follows: the optimal crack extension should approach the correct shape (3.11) in the limit $c_{2} \rightarrow 0$. Under this assumption we can easily obtain the value of $k$. Let us write (3.12) in the following way:

$$
w(t)=\frac{\left(6 c_{1} c_{2} i t+k\right)^{2 / 3}-c_{1}^{2}}{4 c_{1} c_{2}}
$$

To have a well defined limit path we must cancel the numerator for $c_{2}=0$. This gives $k^{2 / 3}=c_{1}^{2}$ and then $k=\left|c_{1}\right|^{3}$ as in (3.13). With this value of $k$ we can now take the limit in (3.14) for $c_{2} \rightarrow 0$ :

$$
w(t)=\lim _{c_{2} \rightarrow 0} \frac{\left(6 c_{1} c_{2} i t+\left|c_{1}\right|^{3}\right)^{2 / 3}-c_{1}^{2}}{4 c_{1} c_{2}}=\frac{2}{3} \frac{6 c_{1} i t\left(\left|c_{1}\right|^{3}\right)^{-1 / 3}}{4 c_{1}}=\frac{i t}{\left|c_{1}\right|},
$$

being the same as the one predicted for $c_{2}=0$ in (3.11).

Notice that the omitted set

$$
w(t)=\frac{\left(6 c_{1} c_{2} i t+\left|c_{1}\right|^{3}\right)^{2 / 3}-c_{1}^{2}}{4 c_{1} c_{2}},
$$

parameterized by $t$, is symmetric with respect to the real axis. Therefore we can find an optimal $F$ satisfying the condition of symmetry (1) and the hydrodynamic expansion (2) in Definition 2. Applying the map $w \rightarrow-w^{2}$ we are able to obtain the shape of the starting crack in the original domain. Taking a Taylor expansion of $-w(t)^{2}$ with respect to $t$ around $t=0$, we have that

$$
-\left(\frac{\left(6 c_{1} c_{2} i t+\left|c_{1}\right|^{3}\right)^{2 / 3}-c_{1}^{2}}{4 c_{1} c_{2}}\right)^{2}=\frac{t^{2}}{c_{1}^{2}}-2 i \frac{c_{2}}{c_{1}} \frac{t^{3}}{\left|c_{1}\right|^{3}}+O\left(t^{4}\right),
$$

which gives the following shape for the starting crack:

$$
x_{1} \approx \frac{t^{2}}{c_{1}^{2}} \Rightarrow \sqrt{x_{1}} \approx \frac{t}{\left|c_{1}\right|}, \quad x_{2} \approx-2 \frac{c_{2}}{c_{1}} x_{1}^{3 / 2} .
$$

The result given by (3.15) is the same qualitative behaviour that the one obtained for a limit of straight kinks of vanishing lengths in [21]. It shows how the shape of the growing crack depends on the local field and deviates from the straight line following the path which releases the maximum amount of energy. In spite of that, (3.15) may not be correct from the physical point of view. Inside the family $\mathcal{F}_{R}$ we are considering crack extensions of different lengths, which means that they involve a different amount of energy dissipated. Therefore, the shape given by this optimal path is somehow artificial because it is not taking into account the full physical picture.

In the following Section we will consider as trial paths the family of shapes given by:

$$
x_{2}=c x_{1}^{3 / 2},
$$

with the complete energy and dissipation terms. This will be carried easily with the help of the Löwner approach for univalent maps, and some available explicit solutions. 


\section{Löwner evolutions.}

The results of the previous Section show that, among all the shapes contained in the family $\mathcal{F}_{R}$, the one optimizing the energy release rate is given by a straight vertical slit (cf. Section 3.4) starting from the origin. When we considered the full energy functional (3.7), Schiffer's method suggested the path (3.16) as the best strategy, but we needed some ad-hoc assumptions to justify this result.

In this Section we will take (3.16) as a family of trial paths, but taking into account the full physical picture, ie. with the dissipation term, following Fourier's inequality (1.4). For that purpose we will take advantage of some recent explicit solutions to the so-called Löwner equation (cf. [14]). We begin with a short exposition of the Löwner approach following [17], and we suggest the reading of the Introduction in [14] for a quick excursion into the subject.

Consider again Figure 3 with the map $F$ normalized as in 2) of Definition 2 and the interval $I$ such that $F(I)=\Delta \Gamma$. Now let us assume that $\Delta \Gamma$ is parameterized in a one to one way by $\gamma: 0 \leq t \leq T \mapsto \mathbb{H}$. We denote the evolution of the tip as $\gamma(t)$ and the whole set of the crack extension up to time $t$ by $\gamma[0, t]$. We will impose that all the trial slits depart from the origin, ie. $\gamma(0)=0$. The parameter $t$ may be selected in such a way that the coefficient $b_{1}(t)$ for each normalized map $F_{t}: \mathbb{H} \mapsto \mathbb{H} \backslash \gamma[0, t]$ is $-2 t$ :

$$
F_{t}(z)=z-\frac{2 t}{z}+\frac{b_{2}(t)}{z^{2}}+O\left(1 / z^{3}\right)
$$

We define $\xi_{t}$ as $F_{t}^{-1}(\gamma(t))$. Notice that $\xi_{t} \in \mathbb{R}$. Under this conditions it can be shown (cf. [17]) that $F_{t}$ satisfies the following differential equation, known as the chordal Löwner equation:

$$
\partial_{t} F_{t}(z)=-\frac{2 \partial_{z} F_{t}(z)}{z-\xi_{t}}, \quad F_{0}(z)=z .
$$

Conversely, given a function $\xi_{t}$ (also called driving force in the mathematical literature!) which is Hölder continous with exponent $1 / 2$ and small norm, equation 4.2 generates slit maps $F_{t}$ (see [19]).

\subsection{Constant forcing}

We start with an interpretation of the result in Section 3.4 in terms of the Löwner equation. In that case we had to minimize the coefficient $b_{1}$ of the map $F$. In terms of (4.1) this means that we have to choose the function $\xi_{t}$ in (4.2) in such a way that $t$ is as big as possible, with the additional constraint that the interval $I_{t}=F_{t}^{-1}(\gamma[0, t])$ should be contained in $[-2 R, 2 R]$. The unique solution given there corresponds to the constant driving force $\xi_{t} \equiv 0$. Equation (4.1) turns out to be:

$$
\partial_{t} F_{t}(z)+\frac{2 \partial_{z} F_{t}(z)}{z}=0, \quad F_{0}(z)=z .
$$

Along the characteristics we must have that

$$
z^{\prime}=\frac{2}{z} \Rightarrow \frac{z^{2}}{2}-\frac{z_{0}^{2}}{2}=2 t
$$

Finally

$$
F_{t}(z)=F_{0}\left(z_{0}\right) \equiv z_{0}=\sqrt{z^{2}-4 t} .
$$

The interval $I$ is given by $[-2 \sqrt{t}, 2 \sqrt{t}]$ and then $t_{\max }=R^{2}$. We have that

$$
F_{t_{\max }}(z) \equiv F(z)=\sqrt{z^{2}-4 R^{2}}
$$

giving the same result as (3.9).

We can now write the energy balance between the Elastic and Dissipation terms following Fourier's inequality (1.4) during critical growth (1.5). The term corresponding to elastic potential energy is given by

$$
\delta E=\frac{\pi}{2} c_{1}^{2} b_{1}\left(F_{t}\right)+o(t)=-\frac{\pi}{2} c_{1}^{2} 2 t+o(t) .
$$


The dissipation is proportional to the length of a straight segment in the true physical plane. The tip moves there following the segment:

$$
x_{1}(t)+i x_{2}(t)=-(2 i \sqrt{t})^{2}=4 t .
$$

The dissipation is then written as

$$
\delta Q=4 \kappa t,
$$

where $\kappa$ is the amount of work per unit length needed to open the crack (see the Introduction). Compatibility in the critical state gives

$$
-\delta E=\delta Q \text { for } t \rightarrow 0^{+} \Rightarrow \pi c_{1}^{2}=4 \kappa .
$$

Then, we can say that

$$
\left|c_{1}\right|=\sqrt{\frac{4 \kappa}{\pi}}
$$

gives the condition for critical equilibrium and Fourier's inequality provides the fact that the crack will grow in the same direction as the one of the initial configuration. This was already obtained in [21] under a more restrictive set of trial paths. As we mentioned there, this is not enough to find the crack evolution shape, since any smooth curve for a strong enough initial field will satisfy this conditions. In order to find more information we will make use of the full energy functional (3.7) and the intuition obtained through the non-physical optimal problems.

\subsection{Linear forcing and Anti-Symmetry principle}

Let us consider now the case of a slit evolution with linear driving force:

$$
\xi_{t}=\lambda t
$$

The Löwner equation (4.2) is then

$$
\partial_{t} F_{t}(z)=-\frac{2 \partial_{z} F_{t}(z)}{z-\lambda t}, \quad F_{0}(z)=z .
$$

In [14], equation (4.4) is solved explicitly and it is easy to show that the asymptotic behaviour of the tip in the physical plane is given by

$$
y=-\frac{\lambda}{3} x^{3 / 2}+o\left(x^{3 / 2}\right) .
$$

This is the same qualitative behaviour as the one obtained in (3.16) through Schiffer's method and is the one predicted in [21] for a limit of little kinks. Our next step is to write the energy expansion in terms of $t$, and show the explicit dependence of the terms on $\lambda$.

We start with the the energy functional (3.7). We must compute the coefficients $b_{1}(t)$ and $b_{2}(t)$ generated by (4.4). Writing for $F_{t}$ :

$$
F_{t}=z+\frac{b_{1}(t)}{z}+\frac{b_{2}(t)}{z^{2}}+\ldots \quad|z|>Z
$$

for some $Z>0$. We have that

$$
\begin{gathered}
\partial_{t} F_{t}=\frac{b_{1}^{\prime}(t)}{z}+\frac{b_{2}^{\prime}(t)}{z^{2}}+\ldots, \\
\partial_{z} F_{t}(z)=1-\frac{b_{1}(t)}{z^{2}}-2 \frac{b_{2}(t)}{z^{3}}+\ldots
\end{gathered}
$$

Using (4.4) we obtain

$$
\begin{aligned}
\frac{b_{1}^{\prime}(t)}{z}+\frac{b_{2}^{\prime}(t)}{z^{2}}+O\left(z^{-3}\right) & =-\frac{2}{z-\lambda t}\left(1-\frac{b_{1}(t)}{z^{2}}-2 \frac{b_{2}(t)}{z^{3}}+O\left(z^{-4}\right)\right) \\
& =-\frac{2}{z}-\frac{2 \lambda t}{z^{2}}+O\left(z^{-3}\right),
\end{aligned}
$$


and therefore

$$
\begin{array}{ll}
b_{1}^{\prime}(t)=-2 & b_{1}(0)=0 \\
b_{2}^{\prime}(t)=-2 \lambda t & b_{2}(0)=0 .
\end{array}
$$

This gives the unique solutions

$$
b_{1}(t)=-2 t, \quad b_{2}(t)=-\lambda t^{2} .
$$

An extension evolving up to "time" $t$ will release an amount of energy (cf. 3.7) given by

$$
\begin{aligned}
\delta E & =\frac{\pi}{2}\left(c_{1}^{2} b_{1}\left(F_{t}\right)+4 c_{1} c_{2} b_{2}\left(F_{t}\right)\right)+O\left(t^{3}\right) \\
& =-\pi c_{1}^{2} t-2 \pi \lambda c_{1} c_{2} t^{2}+O\left(t^{3}\right) .
\end{aligned}
$$

For the dissipation term we must know the evolution of the length in the true physical plane. Let us call $F_{\lambda, t}(z)$ the solution of (4.4) for a given value of $\lambda$. It is easy to show that we can obtain every solution from $F_{1, t}$ by means of a change of scale:

$$
F_{\lambda, t}(z)=\frac{1}{\lambda} F_{1, \lambda^{2} t}(\lambda z) .
$$

Notice that the factor $\frac{1}{\lambda}$ is needed to fit the hydrodynamic normalization. It can also be shown that the trace of singularities $\gamma_{\lambda, t}$ satisfies the following scaling:

$$
\gamma_{\lambda, t}= \begin{cases}\frac{1}{\lambda} \gamma_{1, \lambda^{2} t} & \lambda>0 \\ \frac{1}{\lambda} \gamma_{1, \lambda^{2} t} & \lambda<0 .\end{cases}
$$

On the other hand, the explicit computations done in [14] provide the behaviour of $\gamma_{1, t}$ for $t \rightarrow 0$ :

$$
\gamma_{1, t}=2 i t^{1 / 2}+\frac{2}{3} t-\frac{i}{18} t^{3 / 2}+O\left(t^{2}\right) .
$$

Taking into account the scaling (4.5) of $\gamma_{\lambda, t}$ we have that

$$
\gamma_{\lambda, t}=2 i t^{1 / 2}+\frac{2}{3} \lambda t-\frac{i}{18} \lambda^{2} t^{3 / 2}+O\left(t^{2}\right)
$$

The trial path in the physical plane is given by

$$
-\left(\gamma_{\lambda, t}\right)^{2}=4 t-\frac{8}{3} i \lambda t^{\frac{3}{2}}-\frac{2}{3} \lambda^{2} t^{2}+O\left(t^{5 / 2}\right)
$$

We compute the tangent to this curve

$$
4-4 i \lambda t^{1 / 2}-\frac{4}{3} \lambda^{2} t+O\left(t^{3 / 2}\right),
$$

and the length of the crack extension is given by the expression

$$
l(t)=4 \int_{0}^{t} \sqrt{\left(1-\frac{\lambda^{2}}{3} \tau\right)^{2}+\lambda^{2} \tau+O\left(\tau^{2}\right)} d \tau=4 t+\frac{2 \lambda^{2}}{3} t^{2}+O\left(t^{3}\right) .
$$

We gather the last computations in the following equation:

$$
-\delta E-\delta Q=\left(\pi c_{1}^{2}-4 \kappa\right) t+\left(2 \pi \lambda c_{1} c_{2}-\frac{2 \kappa \lambda^{2}}{3}\right) t^{2}+O\left(t^{3}\right)
$$

The term of order $t$ cancels out due to the critical condition $\left|c_{1}\right|=\sqrt{\frac{4 \kappa}{\pi}}$ in (4.3). In order to satisfy the quasistatic growth condition (1.4) we need the term of order $t^{2}$ to be non-positive. We compute the maximum with respect to the parameter $\lambda$. We obtain

$$
\frac{d}{d \lambda}\left(2 \pi \lambda c_{1} c_{2}-\frac{2 \kappa \lambda^{2}}{3}\right)=0
$$


and then

$$
\lambda=\frac{3 \pi c_{1} c_{2}}{2 \kappa} .
$$

Minus the energy increment for this value of $\lambda$ is

$$
-(\delta E+\delta Q)=\frac{3}{2} \frac{\pi^{2}}{\kappa} c_{1}^{2} c_{2}^{2} t^{2}+O\left(t^{3}\right)>0 \quad \text { for } t \rightarrow 0 .
$$

This means that: if $\left|c_{1}\right|=\sqrt{\frac{4 \kappa}{\pi}}$ and $c_{2} \neq 0$, there exists a trial path violating the quasi-static growth condition (1.4). In other words, there is an instability of second order in the expansion of $\delta E+\delta Q$ (the total energy increment) which allows the energy to go downhill, breaking the equilibrium process. The only way to avoid such a singular behaviour, incompatible with our quasi-static assumption, is a new claim on the local field, that we might call the anti-symmetry principle:

$$
c_{2}=0 \quad \text { for a stable equilibrium configuration. }
$$

As we already mentioned, this condition is quite similar in nature to the so-called symmetry principle for modes I and II of propagation. In that case, the absence of a $K_{\mathrm{II}}$ component implies a completely symmetric field with respect to the crack line. In our case, let us recall the asymptotic expansion around the tip for a fairly smooth portion of the crack near the tip (cf. (1.1) and (2.19)):

$$
u(r, \theta)=k_{0}+k_{1} r^{1 / 2} \sin (\theta / 2)+k_{2} r \cos (\theta)+\ldots
$$

The absence of the $k_{2}=-c_{2}$ term implies that the first two contributions after the constant $k_{0}$ generate anti-symmetric displacement fields with respect to the plane containing the crack $\left(x_{2} \equiv 0\right)$. In other words, the crack should evolve avoiding a symmetric contribution of the field along the line of propagation.

Summing up, the detailed study of the energy landscape variations for different trial paths shows that the two scalar conditions for quasistatic crack propagation in Mode III are given by:

$$
\begin{array}{ll}
k_{1}^{2}=\frac{4 \kappa}{\pi} & \text { critical growth }, \\
k_{2}=0 & \text { quasistatic compatibility },
\end{array}
$$

where $k_{1}$ and $k_{2}$ are obtained in the typical expansion of the displacement around the tip for an out of plane field (1.1).

\section{Concluding remarks}

Löwner evolutions provide a suitable family of trial paths to characterize the path of a crack in quasistatic propagation. The linear driving force show the correct scale to manage the energy released and the dissipation in accordance to Fourier's inequality (1.4).

Condition (4.9) is typical in Linear Elastic Fracture Mechanics. It represents the balance of generalized (or so-called configurational) forces in the direction of propagation. On the other hand, condition (4.10) is more subtle and it is not obtained by first order variations of the energy. The vanishing of the coefficient $k_{2}$ is a free boundary law that penalizes the presence of an instability of second order in the energy landscape around an equilibrium point. It provides a second scalar equation for crack path.

The crack propagation law depends on the coefficients of the asymptotic expansion of the field around the tip. This means that the conditions on the crack path are local. Of course $k_{1}$ and $k_{2}$ depend on the far body-load configuration, but the shape of the crack path will be affected only through the value of the coefficients defining the local field. In other words, any change in the far conditions giving the same values of $k_{1}$ and $k_{2}$ will not affect the behaviour of the path. Notice that no global optimal energy criterion is required: the condition of criticality and second order stability are the main ingredients in this physical picture. 


\section{Acknowledgements}

This research was supported by Spanish project BFM 2004-05634 and it started while the author was visiting the Max Planck Institute for Mathematics in the Sciences, Leipzig, in 2004. I wish to thank Dr. Daniel Faraco who showed me the relationship between slit maps and Löwner equation. This article was finished during a visit to the Institute for Applied Mathematics, University of Bonn, supported by project SFB 611. I would like to acknowledge Prof. Felix Otto for some illuminating discussions.

\section{References}

[1] M. Amestoy, J.B. Leblond (1992), "Crack paths in plane situations II: Detailed form of the expansions of the stress intensity factors", Int. J. Solids Structures Vol. 29, Nr. 4, pp. 465-501.

[2] M. Buliga, "Energy minimizing brittle crack propagation", Journal of Elasticity, 52 (1999), pp. 201-238.

[3] B. Cotterell and J.R. Rice (1980) "Slightly curved or kinked cracks", International Journal of Fracture, Vol. 16, No. 2, pp. 155-169.

[4] R. Courant (1950) Dirichlet's Principle, Conformal Mapping and Minimal Surfaces. Pure and Applied Mathematics Series, Volume III. Interscience, New York.

[5] G. Dal Maso and R. Toader (2002), "A model for the quasi-static growth of brittle fracture: existence and approximation results", Archive for Rational Mechanics and Analysis, 162, No.2, pp.101-135.

[6] P.L. Duren (1983). Univalent Functions, A Series of Comprehensive Studies in Mathematics: 259, Springer-Verlag, New York.

[7] G.A. Francfort and C.J. Larsen (2003), "Existence and convergence for quasi-static evolution in brittle fracture", Communications on Pure and Applied Mathematics, Vol. LVI, pp. 1465-1500.

[8] G.A. Francfort and J.J. Marigo (1998), "Revisiting brittle fracture as an energy minimization problem", Journal of the Mechanics and Physics of Solids, Vol.46, No. 8, pp. 1319-1342.

[9] L.B. Freund, Dynamic Fracture Mechanics, 2d edition. Cambridge University Press, Cambridge (1998).

[10] A. Friedman, B. Hu, J.J.L.Velázquez (2000), "The evolution of the stress intensity factors in the propagation of two dimensional cracks", Euro. Jnl. of Applied Mathematics, Vol.11, pp. 453-471.

[11] R.V. Goldstein and R.L. Salganik (1974). "Brittle fracture of solids with arbitrary cracks", International Journal of Fracture 10, pp. 507.

[12] A.A. Griffith (1920). "The phenomenon of rupture and flow in solids", Phylosophical Transactions of the Royal Society (London) A 221, pp. 163-198.

[13] E.Hohlov, S.D.Howison, C. Huntingford, J.R.Ockendon (1994). "A model for non-smooth free boundaries in Hele-Shaw flows", Quarterly Journal of Mechanics and Applied Mathematics, Vol. 47, Pt. 1, pp. 107-128.

[14] W. Kager, B. Nienhuis and L. Kadanoff (2004) "Exact Solutions for Loewner Evolutions", Journal of Statistical Physics, Vol. 115, Nos. 3/4, May 2004, pp. 805-822.

[15] A.A.Lacey, S.D.Howison, J.R.Ockendon and P.Wilmott (1990) "Irregular morphologies in unstable Hele-Shaw free foundary problems", Quarterly Journal of Mechanics and Applied Mathematics, Vol.43, Pt.3, pp. 387-405.

[16] C. Lanczos (1949). The variational principles of Mechanics, University of Toronto Press. 
[17] G.F.Lawler (2001) "An introduction to the Stochastic Loewner Evolution". Preprint available at http://www.math.cornell.edu/ lawler/papers.html

[18] J.B.Leblond (1989), "Crack paths in plane situations I: General form of the expansion of the stress intensity factors", Int. J. Solids Structures Vol. 25, No. 11, pp. 1311-1325.

[19] D.E.Marshall and S.Rohde (2001) "The Loewner differential equation and slit mappings". Preprint available at http://www.math.washington.edu/ ${ }^{\sim}$ marshall/preprints/preprints.html

[20] Z. Nehari (1975), Conformal Mapping, Dover Publications New York.

[21] G.Oleaga (2004), "On the path of a quasistatic crack in Mode III", Journal of Elasticity, 76, nr.2, pp. 163-189.

[22] J.R.Rice (1968). "Mathematical analysis in the mechanics of fracture", in Fracture, Vol.2, ed. H. Liebowitz. New York: Academic, pp. 191-311.

[23] J.L. Schiff (1993), Normal Families, Springer-Verlag New York.

[24] D.J. Unger (1995) Analytical Fracture Mechanics, Academic Press, San Diego, California.

[25] A.Vasil'ev (2003) "Univalent functions in two-dimensional free boundary problems", Acta Applicandae Mathematicae, 79, pp. 249-280. 